\title{
Increased visceral fat distribution and body composition impact cytokine release syndrome onset and severity after CD19 chimeric antigen receptor T-cell therapy in advanced B-cell malignancies
}

David M. Cordas dos Santos,,$^{1-3^{*}}$ Kai Rejeski, ${ }^{1,3-4^{*}}$ Michael Winkelmann, ${ }^{5}$ Lian Liu, ${ }^{6}$ Paul Trinkner, ${ }^{1,2}$ Sophie Günther, ${ }^{1,2}$ Veit L. Bücklein,,4 Viktoria Blumenberg, ${ }^{1,3-4}$ Christian Schmidt, Wolfgang G. Kunz, ${ }^{5}$ Michael von Bergwelt-Baildon, ${ }^{1,3,6}$ Sebastian Theurich ${ }^{1-3 \#}$ and Marion Subklewe $\mathrm{e}^{1,3-4 \#}$

\begin{abstract}
${ }^{1}$ Department of Medicine III, University Hospital, LMU Munich, Munich; ${ }^{2}$ Cancer- and Immunometabolism Research Group, LMU Gene Center, Munich; ${ }^{3}$ German Cancer Consortium (DKTK), Munich Site, and German Cancer Research Center, Heidelberg; ${ }^{4}$ Laboratory for Translational Cancer Immunology, LMU Gene Center, Munich; ${ }^{5}$ Department of Radiology, University Hospital, LMU Munich and ${ }^{6}$ Comprehensive Cancer Center Munich, LMU Munich, Munich, Germany
\end{abstract}

*DMCDS and KR contributed equally as co-first authors.

"ST and MS contributed equally as co-senior authors.

\author{
Correspondence: M. Subklewe \\ marion.subklewe@med.uni-muenchen.de \\ S. Theurich \\ sebastian.theurich@med.uni-muenchen.de \\ Received: October 13, 2021. \\ Accepted: $\quad$ February 9, 2022. \\ Prepublished: February 17, 2022. \\ https://doi.org/10.3324/haematol.2021.280189 \\ (02022 Ferrata Storti Foundation \\ Published under a CC BY-NC license (c) $(1) \circledast$
}

\begin{abstract}
Chimeric antigen receptor T-cell (CAR-T) therapy is associated with a distinct toxicity profile that includes cytokine release syndrome (CRS) and immune effector cell-associated neurotoxicity syndrome (ICANS). CRS is characterized by the release of pro-inflammatory cytokines such as interleukin 6 (IL-6) and is closely linked to CAR-T expansion and bystander cells like monocytes/macrophages. In other hyperinflammatory states, obesity contributes to inflammatory cascades and acts as a risk factor for disease severity. We aimed to study the influence of anthropometric and body composition (BC) measurements on CAR-T-related immunotoxicity in 64 patients receiving CD19-directed CAR-T for relapsed/refractory Bcell malignancies. Patients with grade $\geq 2$ CRS presented with a significantly higher median body mass index (BMI), waist circumference, waist-to-height ratio (WtHR) and visceral adipose tissue (VAT). These parameters were also found to be associated with an earlier CRS onset. Other adipose deposits and muscle mass did not differ between patients with grade 0-1 CRS versus grade $\geq 2$ CRS. Moreover, BC parameters did not influence ICANS severity or onset. In a multivariate binary logistic regression incorporating known risk factors of immunotoxicity, the factors BMI, waist circumference, WtHR and VAT increased the probability of grade $\geq 2$ CRS. Receiver operating characteristic analyses were utilized to determine optimal discriminatory thresholds for these parameters. Patients above these thresholds displayed markedly increased peak IL-6 levels. Our data imply that increased body composition and VAT in particular represent an additional risk factor for severe and early CRS. These findings carry implications for risk-stratification prior to CD19 CAR-T and may be integrated into established risk models.
\end{abstract}

\section{Introduction}

The advent of chimeric antigen receptor T-cell (CAR-T) therapy as a powerful new class of immunotherapeutic agents has improved outcomes for multiple B-cell malignancies in the relapsed/refractory (R/R) setting. ${ }^{1-4} A$ s these therapies move to the clinical mainstream, understanding the mechanisms that drive the unique spectrum of CART-related immunotoxicity becomes paramount.
Cytokine release syndrome (CRS) represents the most commonly observed adverse event and is characterized by an increase of pro-inflammatory cytokines such as interleukin-6 (IL-6), resulting in endothelial activation and diffuse capillary leakage. ${ }^{5}$ The clinical management of CRS follows a risk-adapted strategy for monitoring and therapy, and the anti-IL-6 receptor antagonist tocilizumab is commercially available to ameliorate symptoms. ${ }^{6}$ Several patient-associated risk factors have been identified that 
facilitate CRS development. These include high tumor burden, concomitant and previous infections, increased Creactive protein (CRP) or ferritin, and thrombocytopenia. ${ }^{7-11}$ While CRS represents a prevalent CAR-T-associated toxicity, the wide-spread adoption of risk-adapted supportive measures has reduced the frequency of its high-grade presentation. Still, the systemic hyperinflammation found in CRS can predispose for the neurological side effects of CAR-T, which remain a major clinical challenge. Immune effector cell-associated neurotoxicity syndrome (ICANS) is found in approximately $20-60 \%$ of CAR-T-treated patients and presents with symptoms ranging from mild confusion to aphasia, seizures, cerebral edema, and potentially death. ${ }^{12-14}$ Patient-associated and dynamic risk factors that predispose for ICANS include prior neurologic diseases and structural impairments, organ dysfunction, older age and severe CRS..$^{15-17}$ High-grade ICANS has also been linked to higher peak levels of systemic cytokines and inflammatory mediatiors (e.g., CRP, ferritin and IL-6), ${ }^{8}$ and a lower median peak absolute monocyte count. ${ }^{18}$

In other hyperinflammatory states including COVID-19 or influenza infections, as well as sepsis, obesity has been demonstrated to be a risk factor for disease severity. ${ }^{19-21}$ Amongst the underlying mechanisms, obesity-associated chronic systemic inflammation, termed metaflammation, has been proposed to enhance an hyperinflammatory immune response, which precedes organ dysfunction. ${ }^{22}$ Mechanistically, macrophages and $\mathrm{CD}^{+} \mathrm{T}$ cells can infiltrate fat deposits turning adipose tissue into an inflammatory endocrine organ secreting pro-inflammatory cytokines such as IL-6, TNF- $\alpha$ and IL-1 $\beta .{ }^{23}$ Various adipose tissue sites differentially contribute to metaflammation: visceral adipose tissue (VAT) in particular plays an important role in metaflammation and obesity-associated pathologies such as type II diabetes and cardiovascular diseases..$^{24,25}$ Epicardial adipose tissue (EAT) has been described as a surrogate marker of VAT and, in turn, as a marker for cardiovascular and metabolic morbidity. ${ }^{26,27}$ In contrast, subcutaneous adipose tissue (SAT) displays a more heterogeneous effect on systemic inflammatory processes. $^{28,29}$

More recently, skeletal muscle has also been recognized as a regulator of immune responses. ${ }^{30,31}$ Skeletal muscle cells modulate immune function by signaling through muscle cell-derived cytokines, termed myokines, cell surface molecules and cell-to-cell interactions. Myokines such as IL-6, IL-7 and IL-15 can modulate CD $8^{+}$T-cell homeostasis and promote survival and proliferation of naïve $\mathrm{T}$ and $\mathrm{B}$ cells. ${ }^{32-35}$ Sarcopenia, which describes the loss of skeletal muscle as a consequence of old age and cancer, is often found in cancer patients and has been linked to immune senescence. ${ }^{30}$

Despite rapidly growing evidence that adipose and muscle tissue play an important role in shaping immune re- sponses, the influence of body composition in the context of CAR-T therapy remains poorly understood. Therefore, we aimed to investigate the impact of body composition on severity and dynamics of CRS and ICANS, as well as serum cytokine levels.

\section{Methods}

\section{Patient cohort}

We performed a retrospective chart review of $R / R$ large B-cell lymphoma (LBCL), B-cell precursor acute lymphoblastic leukemia (BCP-ALL) and mantle cell lymphoma (MCL) patients who underwent CAR-T at the University Hospital of the LMU Munich between 01/2019 until $08 / 2021$. Patients received the commercial CAR-T products Axicabtagene ciloleucel (Axi-cel), Tisagenlecleucel (Tisa-cel), or received KTE-X19 in the compassionate use program for MCL. Patients received lymphodepleting chemotherapy (LDC) with fludarabine and cyclophosphamide according to the manufacturers' instructions. ${ }^{2,3} \mathrm{CRS}$ and ICANS were graded according to the American Society for Transplantation and Cellular Therapy (ASTCT) consensus criteria. ${ }^{6}$ All patients were closely monitored prospectively for CRS and ICANS from the day of CAR-T transfusion until at least day 21 , or until symptoms resolved. Swimmers plots were generated via day-by-day recording of ASTCT grade according to the treating physicians' discretion (Online Supplementary Figures S1 and S2). Heat maps were generated by cross-sectional analysis of the mean CRS/ICANS grade between days 0-21. Toxicity management followed institutional guidelines. Patients with CRS grade $\geq 2$ and persistent CRS grade 1 ( $>24$ hours) were treated with the anti-IL-6 receptor antagonist tocilizumab. In the absence of concurrent CRS or if ICANS was refractory to anti-cytokine therapy, corticosteroids were initiated for grade $\geq 2$ ICANS. Clinical metadata was obtained with Institutional Review Board approval and included a waiver of informed consent.

\section{Measurements of body composition}

Body composition (BC) analyses were performed using clinical records (weight, height) and computerized tomography (CT) scans (waist, adipose/muscle tissue distribution) obtained prior to CAR-T transfusion. The majority of patients received CT imaging on the day before LDC initiation (median imaging to LDC time -1 day, interquartile range [IQR] -4 to 0 days). Three anthropometric measures were included: body mass index (BMI), waist circumference (waist) and waist-to-height ratio (WtHR). Waist was derived from single CT slices. ${ }^{36} \mathrm{WtHR}$ was calculated by dividing waist by height. For quantification of SAT, VAT and muscle tissue distribution (psoas muscle index [PMI] and skeletal muscle index [SMI]), segmentation analyses of 
single CT slices at lumbar spine 3 (L3) were performed. Muscle indices (PMI, SMI) were calculated by dividing mean muscle area by height. EAT content was quantified by calculating the mean EAT amount at the bottom, middle (4-chamber view) and top (left main coronary artery view) of the heart. Adipose and muscle tissue discrimination was based on predefined Hounsfield units (HU) ranges ( -190 to $-30 \mathrm{HU}$ for SAT, -150 to $-50 \mathrm{HU}$ for VAT, -190 to $-30 \mathrm{HU}$ for EAT, -29 to $+150 \mathrm{HU}$ for PM/SM). ${ }^{36-38}$ Cross-sectional areas of respective tissues were computed for each image. Segmentation analyses were performed with ImageJ and the Slice-O-Matic software package (v5.0, Tomovision, Magog, Quebec, Canada). Waist was measured with ImageJ software (v2.0).

\section{Analysis of cytokine dynamics}

Baseline cytokine levels were determined prior to LDC (e.g., day -5). Peak CRP (mg/dL), IL-6 (pg/mL), and ferritin $(\mu \mathrm{g} / \mathrm{L})$ levels were measured daily from CAR-T transfusion until discharge and on subsequent outpatient visits. The total study time frame was day 0-21. Laboratory measurements were performed according to clinical standard procedures in the clinical laboratory of the University Hospital of the LMU Munich. The temporal analysis of IL-6 $(\mathrm{pg} / \mathrm{mL})$ over time was performed by computing the aggregated median value for each day between days $0-21$. In order to distinguish differences between curves, mixedeffects analysis considering both time and effect size using the restricted maximum likelihood method was performed (GraphPad Prism v9.0).

\section{Statistical analysis}

Patient characteristics, BC and serum parameters analyses were compared using the Mann Whitney test or Student's $t$-test according to data distribution for continuous variables, and the Fisher's exact test and chi-squared test for categorical variables. Continuous variables were reported as median and IQR if not stated otherwise. The area under the curve (AUC) and the 95\% confidence interval $(95 \% \mathrm{Cl})$ of the receiver operating characteristic (ROC) analysis was computed using the predicted probability for the development of CRS grade $\geq 2$. Optimal discriminatory thresholds were determined by optimizing the respective Youden $J$ statistic. Multivariate adjusted analysis was performed as binary logistic regression for the outcome of grade $\geq 2$ CRS. The model included baseline clinical characteristics (e.g., age, costimulatory domain), laboratory parameters (e.g., albumin, platelet count), markers of inflammation (e.g., CRP, ferritin, IL-6), and markers of tumor burden (e.g., LDH, STLV) as candidate predictors. The individual BC parameters (BMI, Waist, WtHR, and VAT) were introduced to the model separately to account for multicollinearity (Online Supplentary Table S6). In order to measure the relationship between two continuous variables, Spearman correlation analyses were used. Significance was defined as $P<0.05$. Statistical analysis was performed using GraphPad Prism v9.0 (GraphPad Software, Inc.) and SPSS v26.0.

\section{Results}

\section{Description of the cohort and the real-world toxicity profile}

Between January 2019 and August 2021, the incidence and clinical severity of CRS and ICANS was assessed in $52 \mathrm{R} / \mathrm{R}$ LBCL, eight MCL and four BCP-ALL patients (Table 1; Figure $1 A)$. These patients were treated with the commercial CD19-specific CAR-T products Axi-cel $(n=22)$, Tisa-cel $(n=$

Table 1. Baseline patient characteristics.

\begin{tabular}{|c|c|}
\hline Characteristic & All Patients $(\mathrm{N}=64)$ \\
\hline $\begin{array}{l}\text { Basic data } \\
\text { Age in years, median (range) } \\
\text { Female patients, N (\%) }\end{array}$ & $\begin{array}{c}61(19-82) \\
23(35.9)\end{array}$ \\
\hline $\begin{array}{l}\text { Disease entities, N (\%) } \\
\text { DLBCL including THRBCL } \\
\text { Transformed aggressive non-Hodgkin } \\
\text { lymphoma (from MCL, FL, CLL, HL) } \\
\text { PMBCL } \\
\text { MCL } \\
\text { B-ALL }\end{array}$ & $\begin{array}{l}33(51.6) \\
16(25) \\
2(3.1) \\
8(12.5) \\
5(7.8)\end{array}$ \\
\hline Lines of prior therapy, median (IQR) & $4(3-5)$ \\
\hline Prior autologous/allogeneic SCT, N (\%) & $24(37.5)$ \\
\hline $\begin{array}{l}\text { Costimulatory domain, } \mathrm{N}(\%) \\
\text { 4-1BB } \\
\text { CD28z }\end{array}$ & $\begin{array}{l}34(53.1) \\
30(46.9)\end{array}$ \\
\hline $\begin{array}{l}\text { CRS, any grade, N (\%) } \\
\text { Grade } 1 \\
\text { Grade } 2 \\
\text { Grade } 3\end{array}$ & $\begin{array}{c}59(92.2) \\
31(48.4) \\
22(34.4) \\
6(9.4)\end{array}$ \\
\hline CRS onset, days (IQR) & $2(1-5)$ \\
\hline CRS duration, days (IQR) & $6(4-9)$ \\
\hline $\begin{array}{l}\text { ICANS. any grade, N (\%) } \\
\text { Grade } 1 \\
\text { Grade } 2 \\
\text { Grade } 3 \\
\text { Grade } 4 \\
\text { Grade } 5\end{array}$ & $\begin{array}{c}28(43.8) \\
13(20.3) \\
7(11) \\
4(6.3) \\
3(6.4) \\
1(2.1)\end{array}$ \\
\hline ICANS onset, days (IQR) & $8(3-9)$ \\
\hline ICANS duration, days (IQR) & $8(4-14)$ \\
\hline $\begin{array}{l}\text { Toxicity management, } \mathrm{N}(\%) \\
\text { Tocilizumab administration } \\
\text { Corticosteroid administration }\end{array}$ & $\begin{array}{c}54(79) \\
24(37.5)\end{array}$ \\
\hline
\end{tabular}

B-ALL: B-cell acute lymphoblastic leukemia; CLL: chronic lymphocytic leukemia; CRS: cytokine release syndrome; DL/PM/THRBCL: diffuse large/T-cell/histocyte-rich/primary mediastinal B-cell lymphoma; FL: follicular lymphoma; HL: Hodgkin lymphoma; ICANS: immune effector cell-associated neurotoxicity syndrome; IQR: interquartile range; $\mathrm{MCL}$ : mantle cell lymphoma; SCT: stem cell transplantation. 
34), or received KTE-X19 under compassionate use for $\operatorname{MCL}(n=8)$. All transfused patients were included in the analysis. The median BMI was $23.5 \mathrm{~kg} / \mathrm{m}^{2}$ (IQR 21.6-26.7 $\mathrm{kg} / \mathrm{m}^{2}$ ), and the majority of patients displayed a normal $\mathrm{BMI}$ according to World Health Organization criteria (66\%, Figure 1B). Pre-LDC CT imaging studies were utilized to calculate individual SAT, VAT, EAT values as well as skeletal muscle values with respective indices (PM/I, SM/I) (Figure 1C).

CRS of any grade was observed in 59 (92.2\%) patients, nearly half of which developed grade $\geq 2$ CRS (Figure 2A; Table 1). Median CRS onset was 2 days (IQR 1-5) and lasted a median of 6 days (IQR 4-9) (Figure 2B). ICANS was observed in 28 patients (43.8\%), though only eight patients (14.8\%) developed grade $\geq 2$ ICANS (Figure 2A). Median ICANS onset was 8 days (IQR 3-9) and the median ICANS duration was 8 days (IQR 4-14) (Figure 2C). We found that patients that developed more severe immunotoxicity often displayed an earlier onset of toxicity (Online Supplementary Figure S1 and S2). Life-threatening (grade 4) and fatal (grade 5, 1 patient) ICANS only occurred in patients with grade $\geq 2$ CRS (Online Supplementary Figure $S 3 A$ ). In line with this finding, the median CRS grade was higher in patients with grade $\geq 2$ neurotoxicity (median CRS grade 2 vs. $1, P=0.07$, Online Supplementary Figure $S 3 B$ ). These data are consistent with the known role of CRS as a risk factor of neuroinflammation. ${ }^{16,17}$ Across all patients, 79\% (54/64) received IL-6 receptor blockade with tocilizumab, while $37.5 \%(24 / 64)$ were treated with corticosteroids. When comparing the grade $0-1$ versus grade $\geq 2$ CRS and ICANS groups, baseline markers of tumor burden (e.g., LDH, mean tumor volume), inflammation (e.g., CRP, ferritin), and endothelial dysfunction (e.g., platelet count) were balanced between cohorts (Online Supplementary Table S1). Temporally, CRS predominantly occurred in week 1, while ICANS most commonly occurred in week 2 (Figure 2D). In-
A

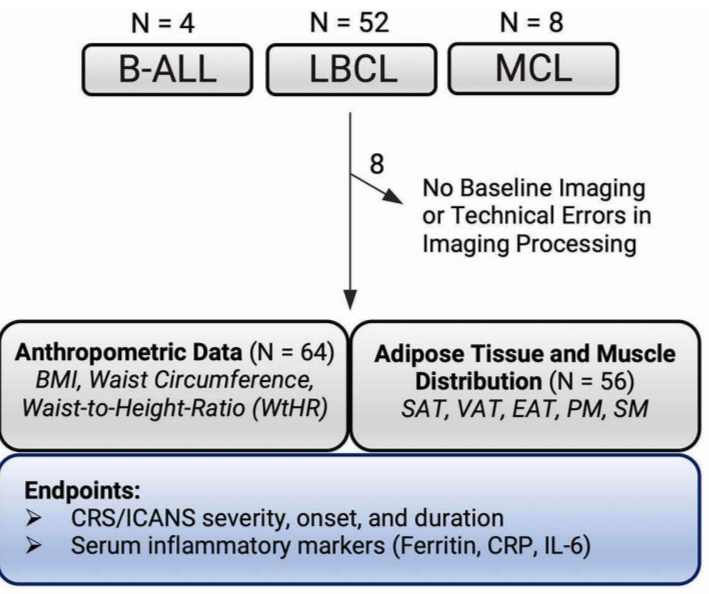

B

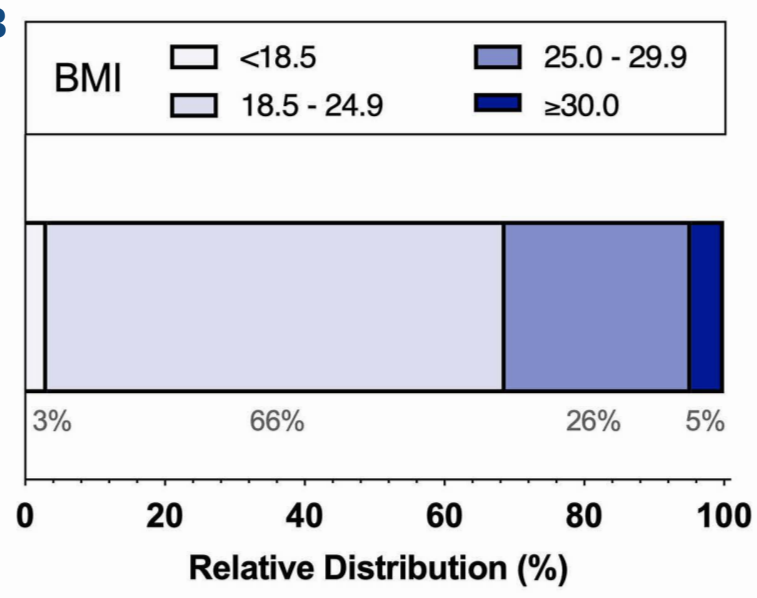

C
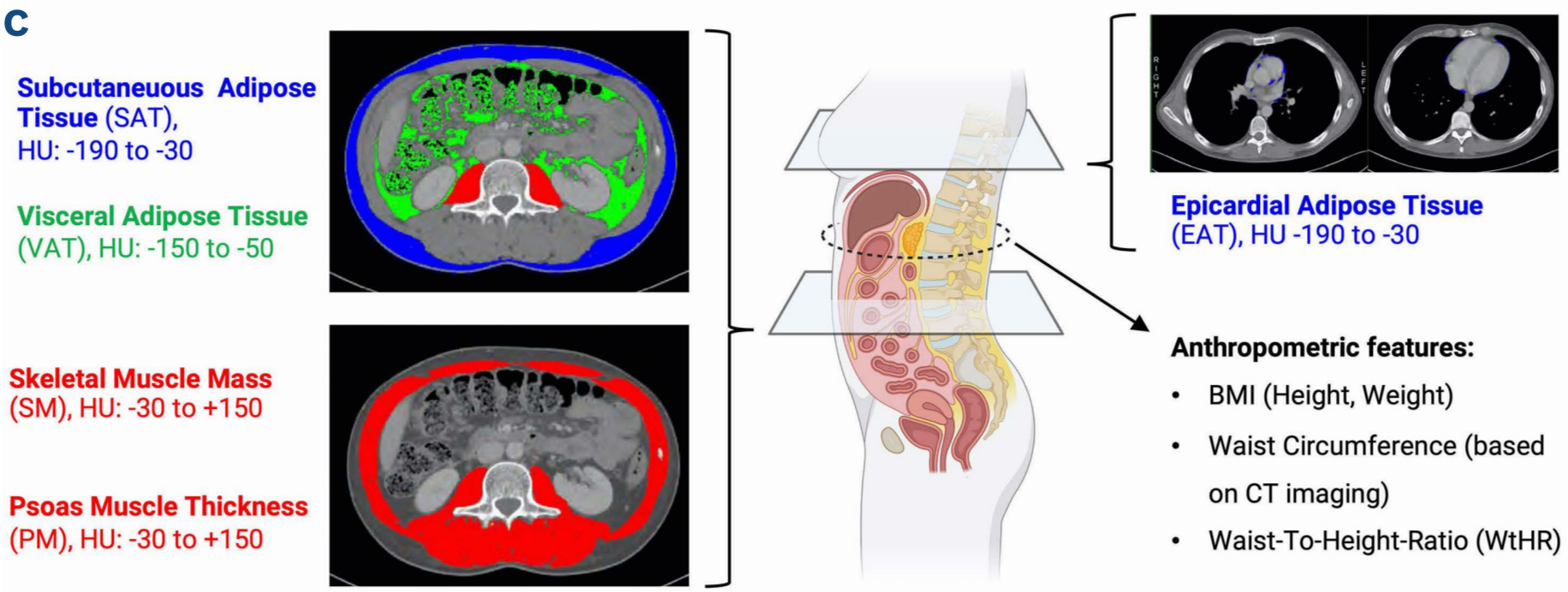

Figure. 1 Measurement of prechimeric antigen receptor T-cell body composition and fat deposition. (A) Cohort description; the primary endpoint was cytokine release syndrome (CRS)/immune effector cell-associated neurotoxicity syndrome (ICANS) severity, onset, and duration; peak cytokine levels represented the exploratory endpoint. Adipose tissue and muscle distribution at baseline (= prior to lymphodepleting chemotherapy [LDC]) could not be determined in 8 patients due to lack of appropriate imaging modalities. Anthropometric data was collected for the entire study cohort $(n=64)$. (B) Relative distribution of body mass index (BMI) according to the 2019 World Health Organization criteria (underweight $<18.5 \mathrm{~kg} / \mathrm{m}^{2}$, normal weight $18.5-24.9 \mathrm{~kg} / \mathrm{m}^{2}$, overweight $25-29.9 \mathrm{~kg} / \mathrm{m}^{2}$, obese $>30 \mathrm{~kg} / \mathrm{m}^{2}$ ). (C) Pretreatment computerized tomography (CT) images were utilized to measure subcutaneous (SAT), epicardial (EAT) and visceral (VAT) adipose tissue deposits, as well as the psoas muscle (PM) thickness and skeletal muscle (SM) mass. SAT, VAT, PM and SM segmentation analyses were performed at lumbar spine 3 level. EAT content was quantified by calculating the mean EAT amount at the bottom, middle (4-chamber view) and top (left main coronary artery view) of the heart. Hounsfield units (HU) describing radiodensity of the respective tissue types are indicated. Anthropometric data included BMI, waist circumference (Waist), and waist-to-height ratio (WtHR). 

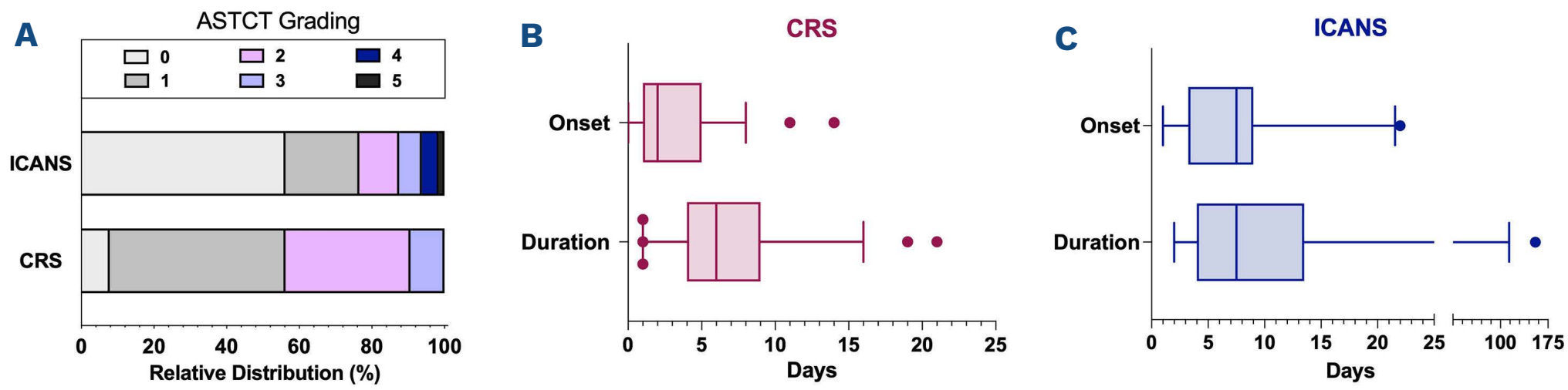

D

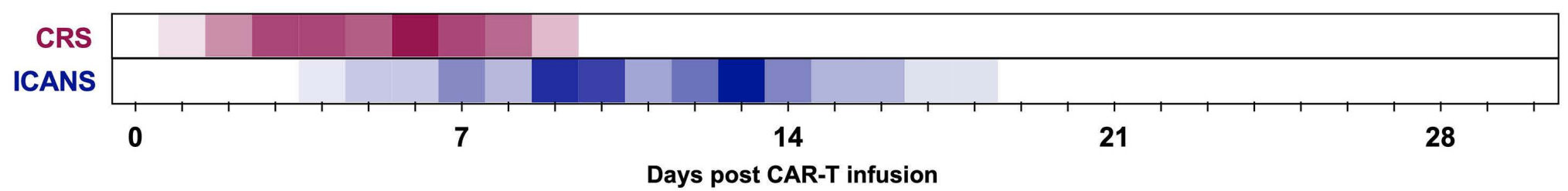

E
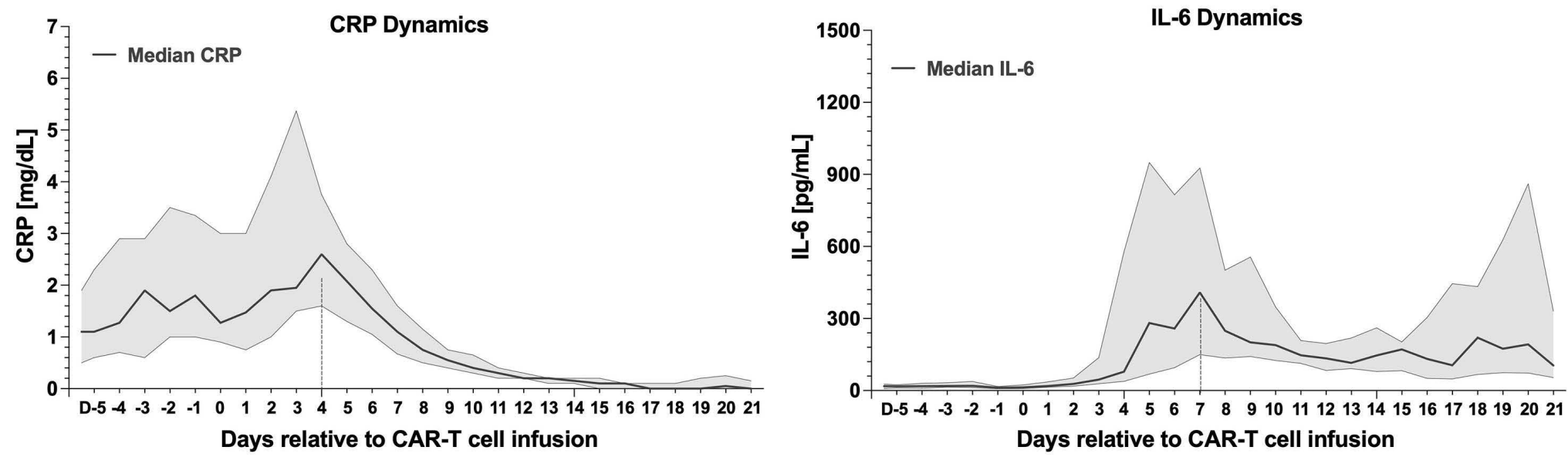

Figure 2. Incidence, onset, and duration of cytokine release syndrome and immune effector cell-associated neurotoxicity syndrome in the study cohort. (A) Relative distribution of cytokine release syndrome (CRS) and immune effector cell associated neurotoxicity syndrome (ICANS) in the studied patient cohort $(n=64)$. Grading was performed according to American Society for Transplantation and Cellular Therapy criteria (Lee et al., BBMT 2019) with color-coding based on severity. (B to C) Box plot displaying median onset and duration of CRS (B) and ICANS (C) in days. The median is reflected by a vertical line, the box displays the interquartile range, whiskers indicate the 95\% confidence interval (CI) of the median. (D) Heatmap outlining the day-by-day time course of CRS (red) and ICANS (blue) after chimeric antigen receptor T-cell (CAR-T) infusion (day 0 [D0]). Darker color indicates higher mean CRS or ICANS grade. (E) Aggregated median C-reactive protein (CRP) (left) and interleukin 6 (IL-6) (right) values over time relative to CAR-T infusion (DO). Light shading depicts the $95 \% \mathrm{Cl}$ of the median for each time point.

flammatory marker profiles revealed a two-phase pattern for IL-6 serum levels with an early peak on day 4 and a second peak observed between days 19-21 (Figure 2F). On the other hand, CRP levels were elevated at baseline, likely reflecting underlying tumor-mediated inflammation. ${ }^{39}$ They reached a peak at day 4 and normalized by day 10 after CAR-T cell transfusion. Overall, these data indicate a representative toxicity profile in our patient cohort.

\section{Body mass index, waist circumference, waist-to-height ratio and visceral adipose tissue are associated with severe cytokine release syndrome}

Previous studies have revealed a link between BC and systemic inflammatory disorders. ${ }^{22}$ We therefore analyzed the distribution of BC parameters in patients with grade 0-1 CRS versus grade $\geq 2$ CRS.

In terms of anthropometric features, we found that the median BMI, waist circumference, and WtHR were signifi- cantly elevated in patients with grade $\geq 2$ CRS (Figure 3; Online Supplementary Table S2). The median BMI was 24.3 $\mathrm{kg} / \mathrm{m}^{2}$ in patients with grade $\geq 2$ CRS compared to 22.9 $\mathrm{kg} / \mathrm{m}^{2}$ in patients with grade 0-1 CRS ( $P=0.01$, Figure 3A). Of the different adipose tissue types, visceral fat deposits in particular were significantly different between the CRS severity groups (127 $\mathrm{cm}^{2}$ vs. $91 \mathrm{~cm}^{2}, P=0.048$, Figure 3D). While a trend was observed for a higher SAT in grade $\geq 2$ CRS, this did not reach statistical significance $\left(169 \mathrm{~cm}^{2} \mathrm{vs}\right.$. $\left.141 \mathrm{~cm}^{2}, P=0.054\right)$. EAT and muscle mass distribution did not differ between CRS severity cohorts (Online Supplementary Table S2).

Next, we performed ROC analyses to determine optimal discriminatory thresholds for each parameter that reached statistical significance (Online supplementary Figure S4) and calculated the concomitant odds ratios (OR) for the outcome grade $\geq 2$ CRS (Table 2). The observed odds for developing grade $\geq 2$ CRS were signifi- 
cantly higher for patients with a BMI $\geq 27.05 \mathrm{~kg} / \mathrm{m}^{2}$ $(P=0.007)$, waist $\geq 99.23 \mathrm{~cm}(P=0.04)$, WtHR $\geq 0.594 \mathrm{~cm} / \mathrm{m}^{2}$ $(P=0.01)$ and VAT $\geq 144.3 \mathrm{~cm}^{2}(P=0.02)$. The AUC of the ROC curve ranged between 0.63 to 0.67 . Importantly, previously identified CRS risk factors such as age, tumor burden and certain laboratory parameters ${ }^{7-11}$ were distributed equally between patient cohorts based on the defined thresholds for BMI, waist, WtHR and VAT (Online Supplementary Table S3). On multivariate adjusted analysis, we confirmed that these four BC parameters represent independent risk factors of grade $\geq 2$ CRS (Table 3 ). With adjusted odds ratios ranging from 1.15 to 1.37 , they signficantly increased the probability of grade $\geq 2$ CRS. Collectively, these findings suggest a contributing role of visceral adipose tissue for CRS severity.

Body mass index, waist, waist-to-height ratio and visceral adipose tissue correlate with cytokine release syndrome dynamics

In addition to CRS severity, we analyzed the impact of anthropometric and BC measurements on CRS dynamics. Linear regression analyses revealed significant negative correlations between CRS onset and BMI, waist, WtHR, and VAT (Figure 3B; Online Supplementary Table S4). Ac-
A

A

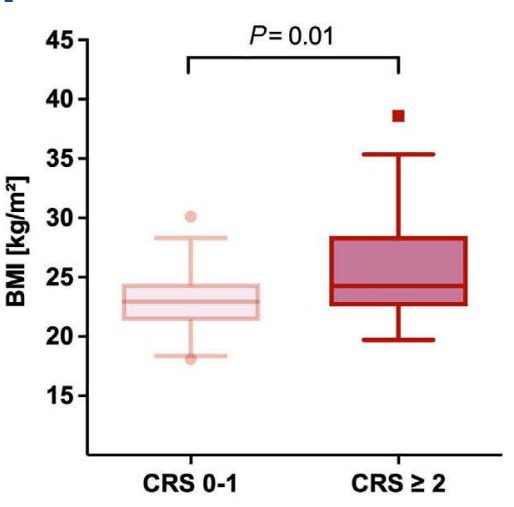

B

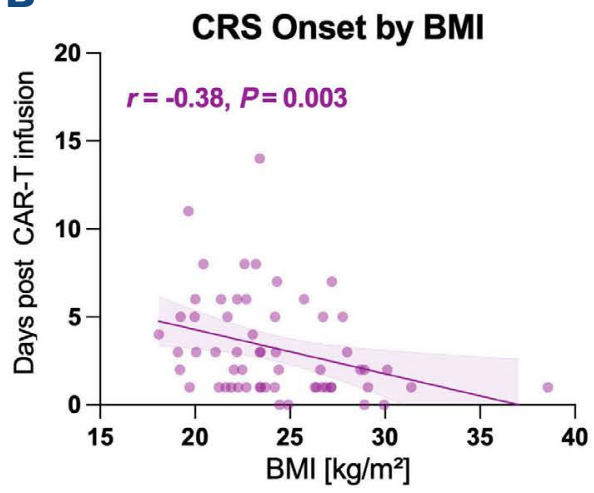

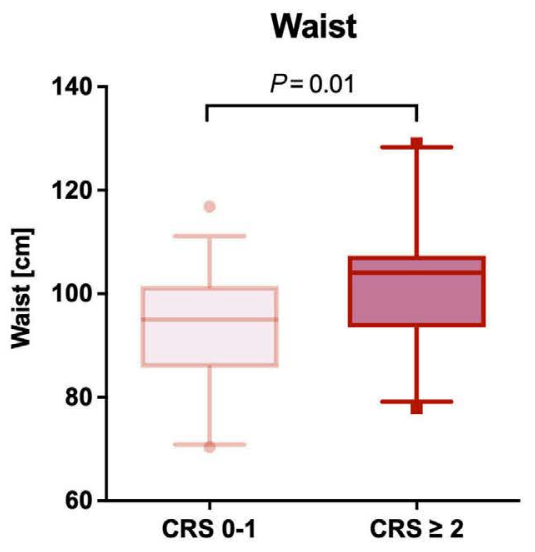

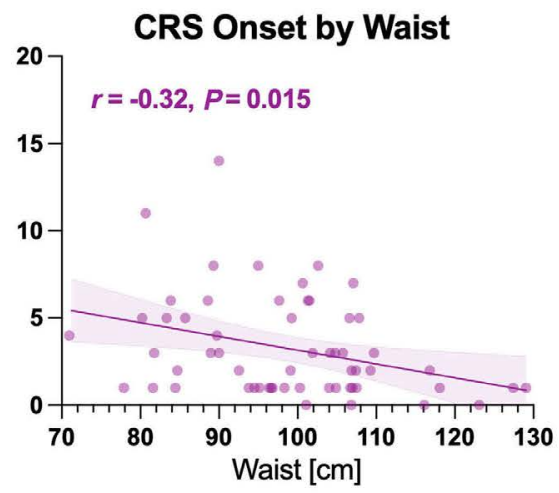

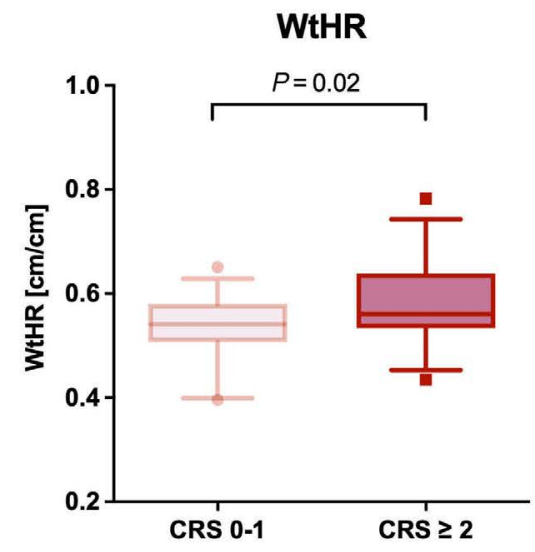
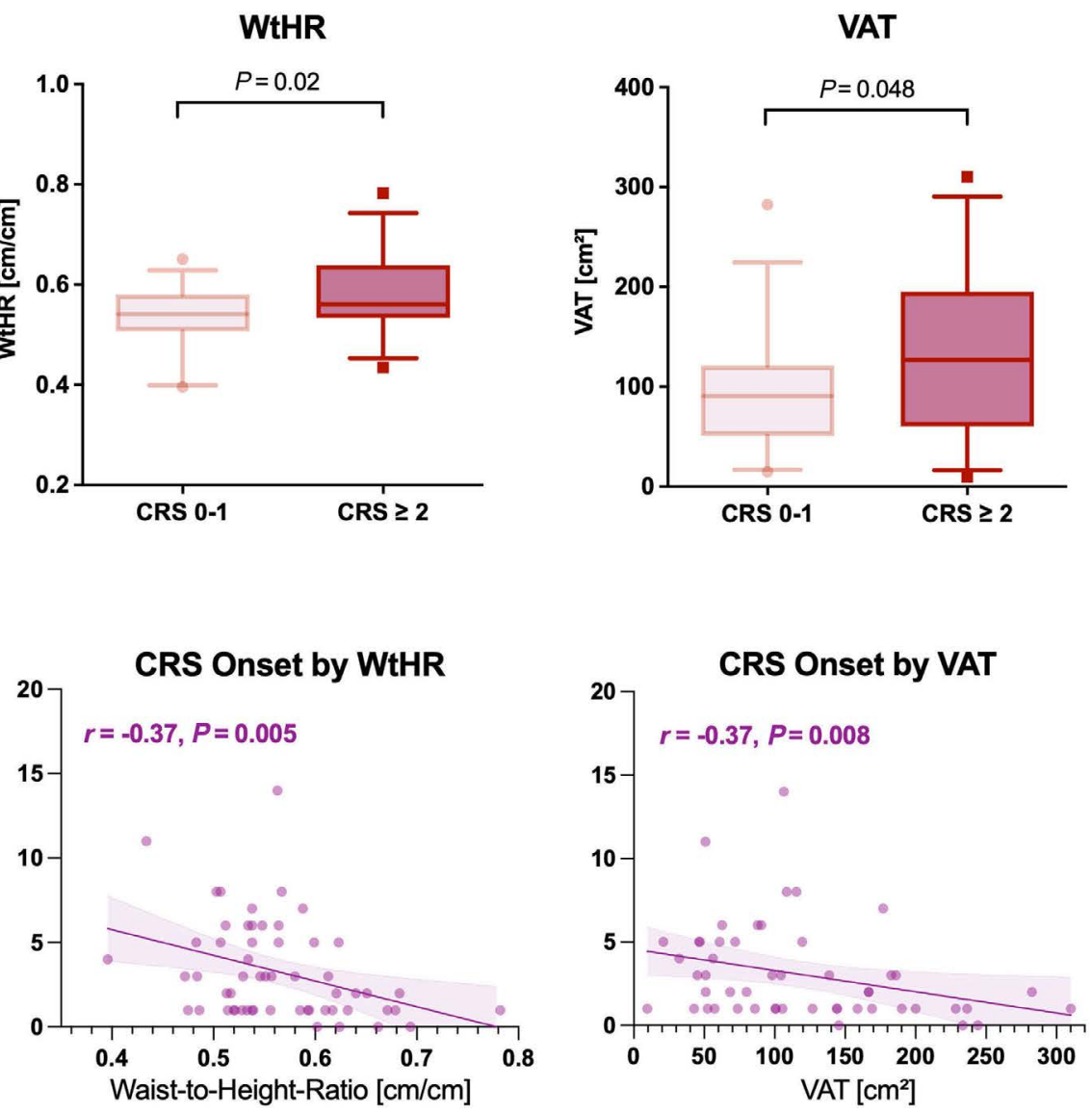

Figure 3. Body mass index and metabolic high-risk adipose tissue are associated with more severe cytokine release syndrome and early cytokine release syndrome onset. (A) Box plots comparing body mass index (BMI), waist circumference (Waist), waistto-height ratio (WtHR), and visceral adipose tissue (VAT) by cytokine release syndrome (CRS) severity. Higher-grade CRS defined as American Society for Transplantation and Cellular Therapy grade $\geq 2$, non-severe CRS as grade $0-1$. The box indicates the interquartile range with the horizontal line describing the median, and whiskers denoting the $95 \%$ confidence interval [Cl] of the median. (B) Univariate linear regression analysis comparing BMI, Waist, WtHR, and VAT (from left to right) vs. CRS onset (purple) in days. The graphical inset depicts the Spearman correlation coefficient with a positive r-value indicating a positive correlation and a negative $r$-value indicating a negative correlation, as well as the respective $P$-value. The $95 \% \mathrm{Cl}$ bands of the best-fit line from the simple linear regression are shown in light shading.

Table 2. Determination of discriminatory thresholds and odds ratios based on receiver operating characteristic analysis computed for predicted probability of grade $\geq 2$ cytokine release syndrome.

\begin{tabular}{|l|c|c|c|c|}
\hline $\begin{array}{l}\text { Body composition } \\
\text { parameter }\end{array}$ & $\begin{array}{c}\text { Discriminatory } \\
\text { threshold }\end{array}$ & AUC & P-Value AUC & OR (95\% Cl) \\
\hline BMI, $\mathrm{kg} / \mathrm{m}^{2}$ & 27.05 & 0.66 & 0.03 & $6.11(1.7-22.1)$ \\
\hline Waist, $\mathrm{cm}$ & 99.23 & 0.67 & 0.02 & $2.96(1.03-8.29)$ \\
\hline WtHR, cm/m ${ }^{2}$ & 0.5935 & 0.65 & 0.04 & $4.06(1.4-11.3)$ \\
\hline VAT, $\mathrm{cm}^{2}$ & 144.3 & 0.63 & 0.09 & $4.8(1.4-15.2)$ \\
\hline
\end{tabular}

95\% CI: 95\% confidence interval; AUC: area under curve; BMI: body mass index; CRS: cytokine release syndrome; OR: odds ratio; VAT: visceral adipose tissue; WtHR: waist-to-height ratio 
cordingly, BMI ${ }^{\text {high }}\left(\geq 27.05 \mathrm{~kg} / \mathrm{m}^{2}\right)$ patients exhibited an earlier median CRS onset compared to their BMI ${ }^{\text {low }}$ counterparts (day 1 vs. day 3, $P=0.05$, Online Supplementary Figure S5). Earlier CRS onset was also noted for WtHR and VAT (Online Supplementary Figure S5). On the other hand, anthropometric and BC parameters did not significantly correlate with CRS duration (Online Supplementary Table S4). These data may reflect the aggressive and early CRS management in this patient cohort, as 91.5\% (54/59) of patients with CRS received tocilizumab after a median of 4 days. In summary, adipose tissue parameters were associated with an earlier CRS onset but not duration.

\section{Sarcopenia and body composition are neither associated with immune effector cell-associated neurotoxicity syndrome severity nor dynamics}

In addition to adipose tissue distribution analyses, whole skeletal muscle mass and psoas muscle mass with respective indices (SMI, PMI) were measured for all patients. Twenty-four patients were classified as sarcopenic corrected for sex and BMI. Distribution of sarcopenia and muscle masses (SMI, PMI) did not differ between grade $\geq 2$ CRS and grade 0-1 CRS patients (Online Supplementary Table S2). Moreover, muscle parameters did not affect CRS onset or CRS duration. Since CRS represents a risk factor for ICANS, ${ }^{16,17}$ we also investigated the influence of $B C$ parameters on severity and dynamics of ICANS. However, none of the measured BC parameters differed between patients with grade 0-1 ICANS versus grade $\geq 2$ ICANS nor did they correlate with either ICANS onset or ICANS duration (Online Supplementary Tables S2 and S5).

\section{Adipose tissue correlates with peak IL-6 levels and IL-6 dynamics}

In order to better understand the potential pathomechan-

Table 3. Body mass index, waist, waist-to-height ratio and visceral adipose tissue represent independent risk factors for grade $\geq 2$ cytokine release syndrome in a multivariate logistic regression analyses including previously described risk factors.

\begin{tabular}{|l|c|c|c|}
\hline Body composition parameter & OR & $95 \%$ CI & $P$-Value \\
\hline BMI, kg/m² & 1.37 & $1.02-1.83$ & 0.04 \\
\hline Waist, cm & 1.16 & $1.04-1.29$ & 0.009 \\
\hline WtHR, cm/m² & 1.24 & $1.05-1.46$ & 0.01 \\
\hline VAT, $\mathrm{cm}^{2}$ & 1.15 & $1.02-1.3$ & 0.02 \\
\hline
\end{tabular}

Odds ratios were calculated based on four separate multivariate logistic regressions for each of the body composition parameters. Complete model parameters and estimates are shown in the Online Supplementary Table S6. 95\% Cl: 95\% confidence interval; AUC: area under curve; BMI: body mass index; CRS: cytokine release syndrome; OR: odds ratio; VAT: visceral adipose tissue; WtHR: waist-to-height ratio. isms that may underlie earlier and more severe CRS in patients with increased adipose tissue, we analyzed serum levels of pro-inflammatory markers and their distribution according to the $\mathrm{BC}$ groups.

First, we correlated BC parameters with baseline and peak serum levels of ferritin, CRP and IL-6-revealing an effect for IL-6 in particular (Online Supplementary Figure S6). Interestingly, peak IL-6 levels were significantly increased in patients with elevated waist circumference (3.442 vs. $1.019 \mathrm{pg} / \mathrm{mL}, P=0.008)$, WtHR (3.768 pg/ml vs. $1.385 \mathrm{pg} / \mathrm{mL}$, $P=0.03$ ), and VAT (6.530 pg/ml vs. $1.143 \mathrm{pg} / \mathrm{ml}, P=0.01$ ) (Figure 4A). A comparison of aggregated median IL-6 courses over time (baseline until day 21) further demonstrated that the median time-to-peak IL-6 was earlier in patients with an increased amount of adipose tissue (Figure 4B). In a comparison of curves accounting for both time and effect size, BMI ${ }^{\text {high }}$ patients developed an earlier IL- 6 peak ( 5 days vs. 7 days) and the peak was approximately 5-fold higher than in $\mathrm{BMI}^{\text {low }}$ patients (top left panel, Figure 4B). The observed differences in IL-6 dynamics were especially prominent in patients with excess visceral adipose tissue (peak 11-fold higher, day 4 vs. day $8, P<0.0001$, lower right panel, Figure 4B). Notably, patients rich in adipose tissue developed a second IL-6 peak, which occurred between days 19-21 in the WtHR ${ }^{\text {high }}$ and VAThigh patients (Figure 4B). These results demonstrate that pro-inflammatory cytokines such as IL-6 are elevated in patients with abundant visceral fat.

\section{Discussion}

The CAR-T-related side effects CRS and ICANS represent a novel toxicity category in the $21^{\text {st }}$ century armentarium of cancer therapy. However, their underlying pathomechanisms remain incompletely understood. Here, we present the first comprehensive analysis of immunometabolically relevant tissues and their impact on CRS and ICANS in patients with advanced B-cell malignancies receiving CAR-T. Our data indicate that increased visceral adipose tissue is associated with CRS severity and early CRS onset. On the other hand, skeletal muscle mass measures did not impact CRS or ICANS occurrence.

The finding that visceral adipose tissue deposits drive differences in CRS severity is in line with previous reports demonstrating a strong association of VAT with obesityassociated metaflammation and the development of cardiovascular diseases and diabetes. ${ }^{24,25}$ The 'obesity paradox', meaning the unexpected inverse relationship between excess adipose tissue and immunotherapy efficacy, has been established for immune checkpoint blockade both in preclinical models and in cancer patients. ${ }^{40} 41$ The observed therapeutic benefit in the excess weight population was further enhanced when immune-related ad- 
A
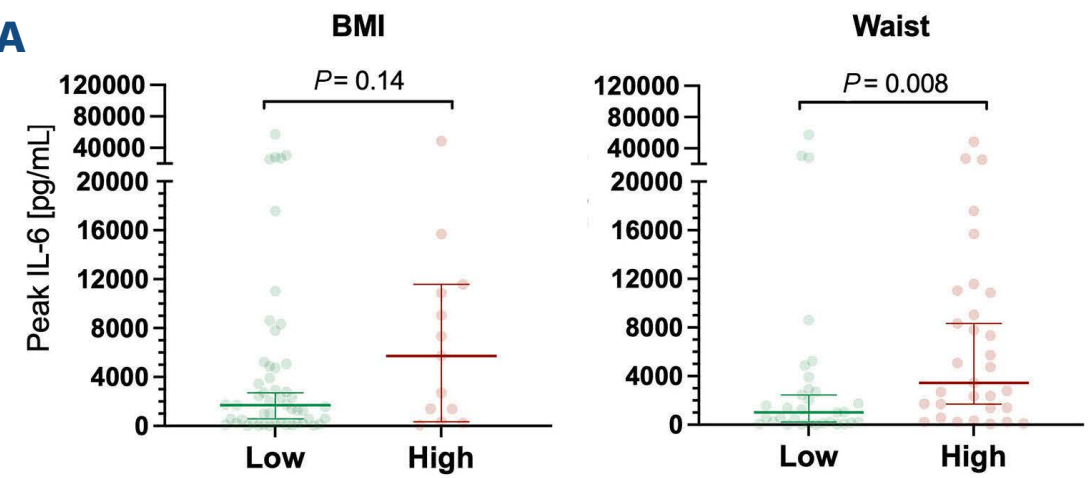

B
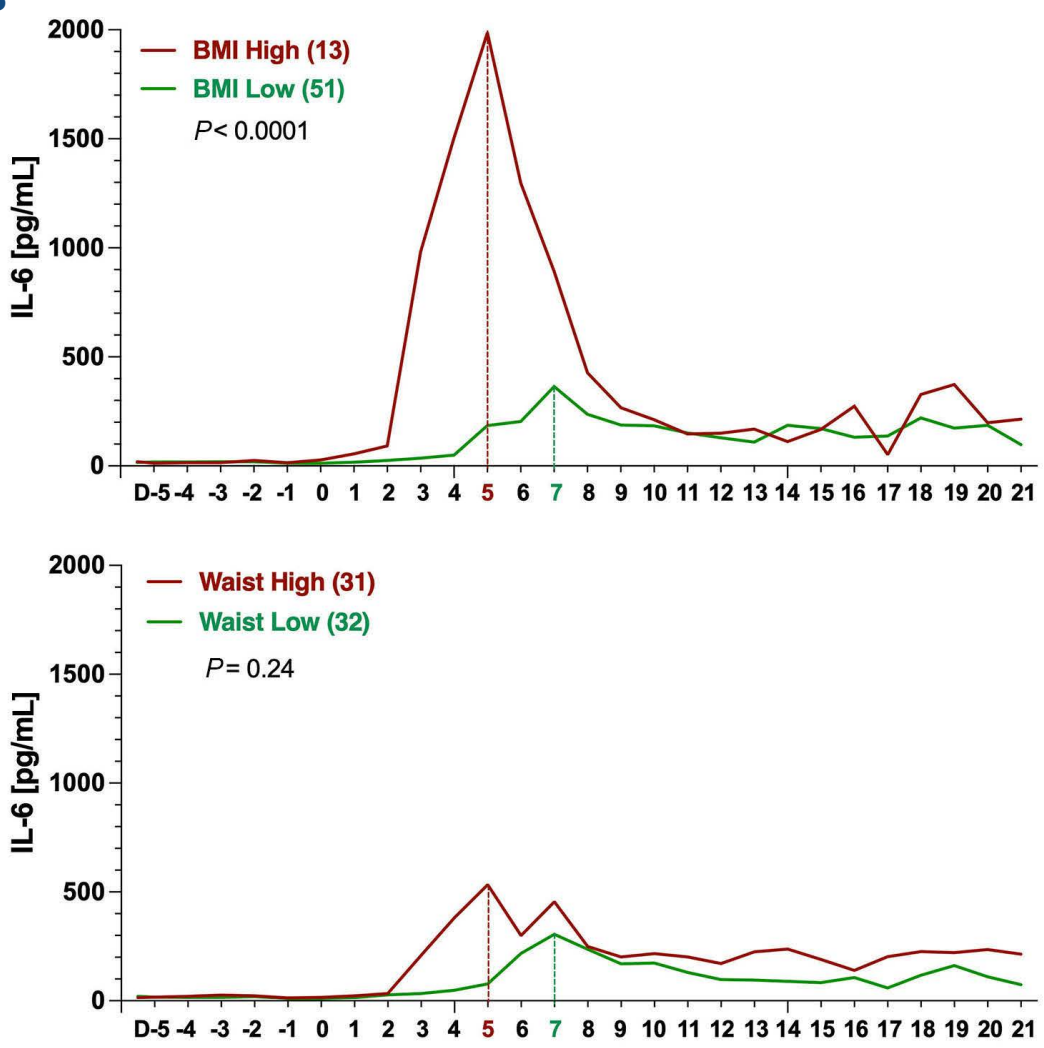
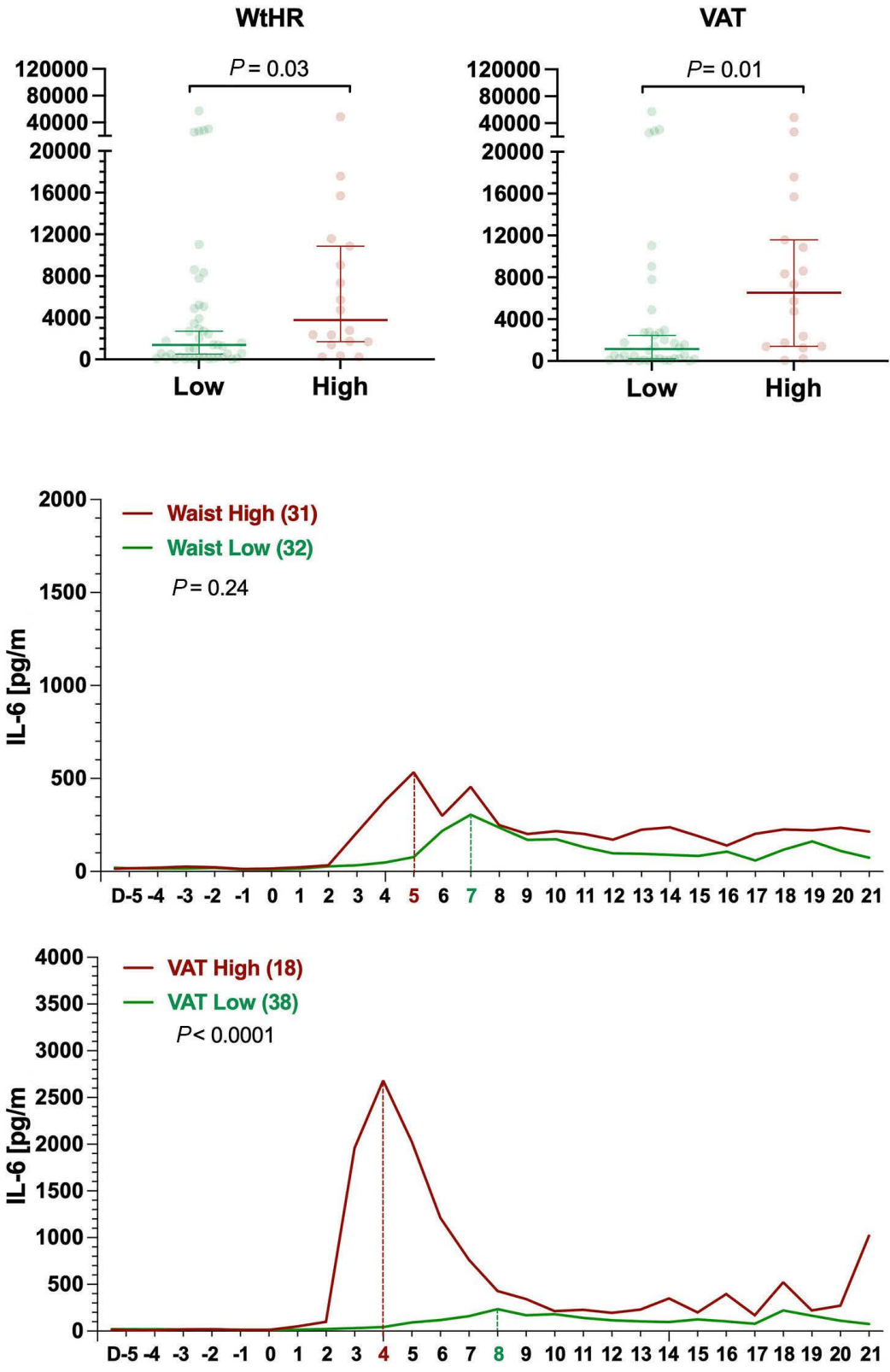

Figure 4. Adipose tissue correlates with peak interleukin 6 and interleukin 6 dynamics. (A) Peak interleukin 6 (IL-6) by body mass index (BMI), waist circumference (Waist), waist-to-height ratio (WtHR), and visceral adipose tissue (VAT) (from left to right). The previously established discriminatory values were used to distinguish high vs. low groups. (B) Aggregated median IL- 6 values over time comparing BMI/Waist/WtHR/VAThigh vs. BMI/Waist/WtHR/VATlow patients. The dotted vertical line indicates the median day of the peak for each group. Significance values were determined by two-way ANOVA considering both time and effect size.

verse events occurred. ${ }^{42}$ However, the impact of overweight and obesity on survival and toxicity has been more mixed in the context of BCL in the pre-CAR-T era. For example, improved outcomes were noted for overweight patients in first-line rituximab-containing chemotherapy regimens, though the opposite was observed in obese patients receiving autologous stem cell transplantation. ${ }^{43,44}$ One study analyzing the impact of body weight on clinical outcomes in R/R LBCL patients receiving CAR-T was negative, though detailed body composition analyses were not performed and only Axi-cel patients were included. ${ }^{45}$ In our study, BC parameters negatively correlated with CRS onset, suggesting that overweight patients harbor a pro-inflammatory environment that predisposes them for earlier and more severe systemic inflammation. This would be consistent with prior studies establishing the role of adipose tissue as an endocrine organ with the potential of amplifying immune responses. ${ }^{22}$ Consistent with this hypothesis, the anthropometric body fat indices and VAT were all associated with elevated peak IL- 6 serum levels. VAThigh patients exhibited not only markedly increased peak IL- 6 levels, but also a shorter time to peak IL-6, mirroring the clinical observation of an earlier CRS onset in VAT ${ }^{\text {high }}$ patients. While we did not observe a link between body composition and CRS duration, this may reflect early tocilizumab administration in this patient cohort, which may have blunted the natural CRS time course. Notably, IL-6 analyses revealed a second peak around day 19-21 for patients with excess body fat. Wei and colleagues propose a conceptual framework wherein a minimal IL- 6 peak in week 3 coincides with the redistribution of CAR T-cells into the periphery in the absence of target cells. ${ }^{46}$ Such redistribution results in the activation of tissue-resident immune cells such as macrophages or neutrophils, and may be potentiated by metaflammation-inherent feedback loops. The second peak also temporally coincides with the burgeoning hematopoetic recovery observed in the third week after CAR-T transfusion. ${ }^{47}$ As diet can impact remodeling of the bone marrow niche and skew hematopoietic stem and 


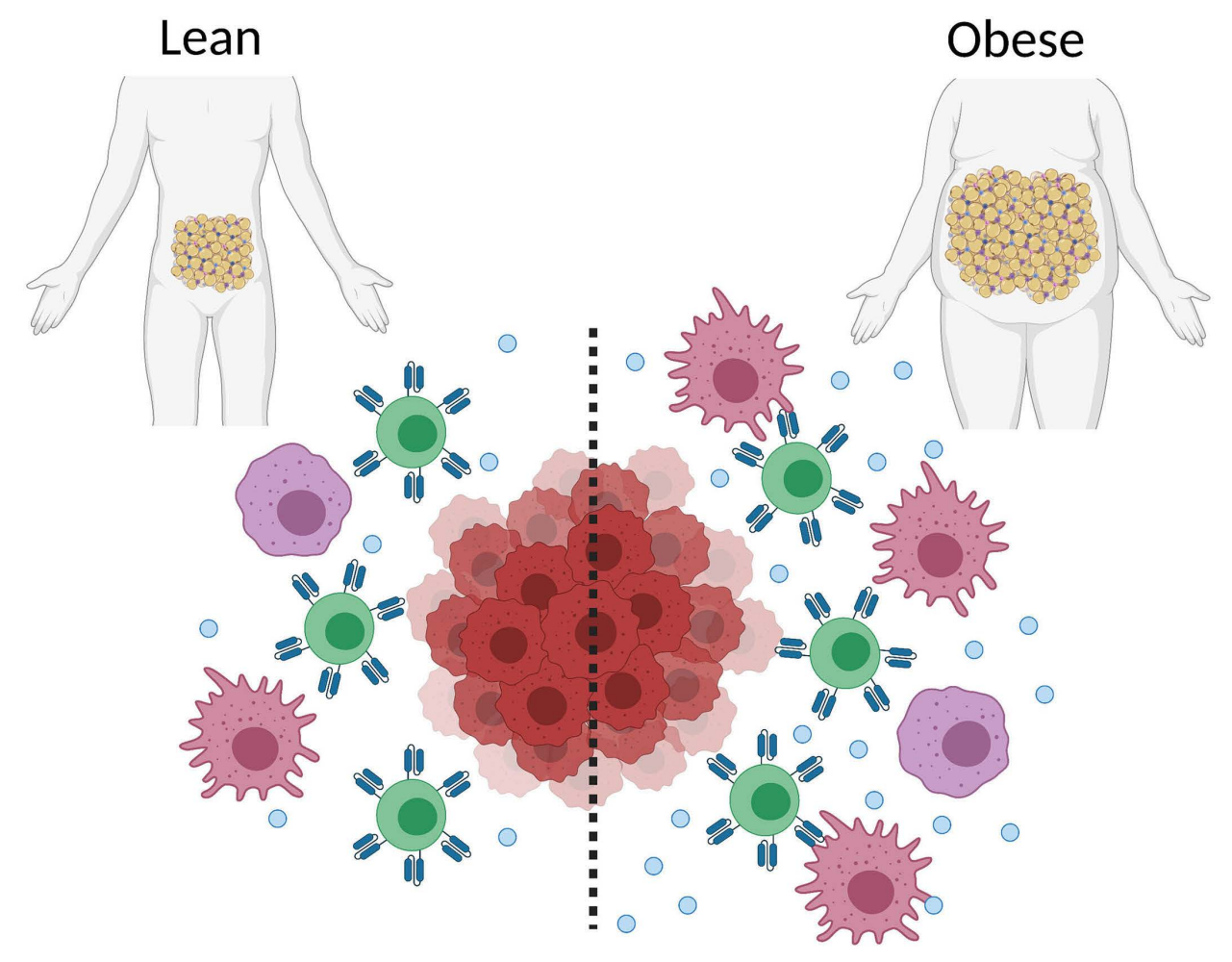

CRS $\downarrow$

CRS $\uparrow$
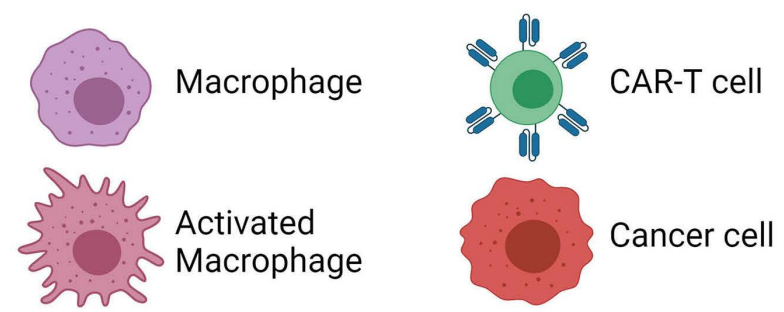

Interleukin 6

Figure 5. Proposed model of meta-inflammation as potentiating factor in cytokine release syndrome pathogenesis.

progenitor cell function, ${ }^{48}$ this may indirectly affect inflammation cascades facilitated by mobilized immune cells - though this remains to be systematically studied in the context of CAR T-cell therapy.

Based on our results, we postulate that elevated visceral adipose tissue mass in overweight or obese CAR-T patients predisposes to more severe and earlier CRS. Furthermore, obesity-associated metaflammation with subsequent elevation of serum IL-6 levels may represent the potential link for the adipose tissue-induced effects (Figure 5). The relationship between obesity and IL-6 in the context of metaflammation has been extensively described, and is predominantly driven via IL- 6 secretion by adipocytes and adipose-tissue macrophages. ${ }^{49-51}$ Surprisingly, we found an association between adipose tissue and CRS in a patient cohort with very few obese patients as defined by World Health Organization criteria (BMI >30 $\left.\mathrm{kg} / \mathrm{m}^{2}, \mathrm{n}=4\right)$. However, this may be explained by the longlasting effect of metaflammation that can be preserved even after weight loss. ${ }^{52}$ Unfortunately, we did not have $\mathrm{BMI}$ data at the time of initial diagnosis for all patients to test this hypothesis. However, we expect that most patients were subject to weight loss due to their disease and treatment. Alternatively, even low amounts of adipose tis- sue may be sufficient to facilitate a pro-immunogenic effect. Nevertheless, further prospective studies, preferably in patient cohorts with a higher percentage of obese patients, are needed to validate the role of overweight and obesity as a risk factor for severe CRS. If validated, anthropometric data like BMI and waist circumference may serve as an attractive auxiliary component in the riskstratification tool box of the CAR-T treating physician due to the easy-to-measure nature of these parameters. For example, acquiring $B C$ data requires only a tape measure and a scale, underlining clinical ease-of-use. This may be especially pertinent for patients who are fit enough to receive CAR-T in an outpatient setting. ${ }^{53}$

In contrast to the interactions between adipose tissue and CRS, we did not find that skeletal muscle mass impacts CRS or ICANS. Although nearly half of our patients were defined as sarcopenic according to the published sarcopenia classifications, ${ }^{54}$ CRS and ICANS severity was equally distributed between sarcopenic and non-sarcopenic patients. There are several potential explanations for this observation. First, patient numbers may have been too small to discern interactions between skeletal muscle and systemic inflammation. Second, previously described interactions between muscle mass and the immune sys- 
tem were performed under stringent physiologic conditions or in the context of aging, ${ }^{55}$ which may be negligible in the context of adoptive immunotherapies like CAR-T. Third, sarcopenia arises from a dysbalance in obesity-associated pro-inflammatory effects leading to muscle catabolism, as well as skeletal muscle-derived anti-inflammatory signaling resulting in muscle anabolism. ${ }^{30}$ The pro-inflammatory effects of adipose tissue and concomitant metaflammation may outweigh the influence of skeletal muscle on immune reactions.

Limitations of this study include its retrospective and single-center design with a limited sample size. Additionally, only a small subset of the cohort was obese, making it difficult to generalize assumptions on obesity as a risk factor for CRS. Importantly, the results of the present study need to be validated in larger patient cohorts across multiple health care systems and institutions. This may lead to the development of a BC-based risk score for CRS in patients receiving CD19 CAR-T. Moreover, we have described optimal discriminatory thresholds for BC parameters, which may be integrated into existing risk models of CAR-T related immunotoxicity to improve diagnostic accuracy and predictive capacity. ${ }^{711,47,56}$ Such enhanced risk models can form the basis of interventional studies exploring how CAR-T toxicity and efficacy are impacted by early and/or prophylactic anti-cytokine therapies (e.g., tocilizumab, anakinra) or corticosteroids. Finally, further mechanistic exploration of (CAR) T-cell expansion and distribution of immune cell phenotpyes in the context of elevated BMI or VAT appears warranted.

Still, these initial data provide evidence that BC and adipose tissue distribution matter in patients receiving $\mathrm{T}$-cell based immune therapies. The finding that patients with an elevated BMI develop earlier and more severe CRS invites future translational research, and underlines how metainflammation may serve as a potentiating force in systemic inflammatory disorders.

\section{Disclosures}

$K R$ has received research funding from Kite/Gilead. VB has received research funding from Kite/Gilead, BMS/Celgene and Janssen and consulted for Kite/Gilead and Novartis. $V L B$ received research funding from BMS/Celgene, Miltenyi and Novartis; received honoraria and travel support from Amgen, Kite/Gilead, Novartis and Pfizer; consulted for Kite/Gilead, BMS/Celgene, Novartis and Pfizer; was part of speakers bureau for Novartis and Pfizer. CS work was funded by Bayer and Kite/Gilead; has received honoraria and travel costs from BMS, Janssen, Kite/Gilead, Novartis and consulted for BMS, Kite/Gilead, Novartis and Takeda. $M B$ received honoraria, was part of the speakers bureau of and consulted for MSD Sharpe \& Dohme, Novartis, Roche, Kite/Gilead, BMS/Celgene, Astellas, Mologen and Miltenyi. ST received honoraria from and consulted for Amgen, BMS/Celgene, GSK, Janssen, Pfizer, Sanofi and Takeda. MS received honoraria, was part of speakers bureau and was funded by Amgen, BMS/Celgene, Gilead, Novartis; she further received funding from Miltenyi, Morphosys, Roche; she received honoraria and research funding from Seattle Genetics, honoraria from Janssen and Pfizer and was part of speakers bureau for Pfizer and Takeda. All other authors have no conflicts of interest to disclose.

\section{Contributions}

$D M C D S, K R$, ST and MS designed the study and developed the concept; DMCDS, KR, MW, LL, PT, SG, VLB, VB, CS, WGK, $M B B, S T$, and MS carried out the research; DMCDS, KR, ST and MS performed the formal analysis and visualized the study; DMCDS, KR and LL developed the methodology; $D M C D S, K R, S T$ and MS wrote the original draft; DMCDS, $K R, M W, L L, V L B, V B, C S, W G K, M B B$, ST and MS revised and edited the manuscript. All authors read and approved the final manuscript.

\section{Acknowledgments}

We are grateful for the support of all patients and the personnel of the LMU University Hospital who supported this work. Figure 1 and 5 were created with BioRender.com.

\section{Funding}

$D M C D S, K R, V B$ received a fellowship from the School of Oncology of the German Cancer Consortium (DKTK). $D M C D S$ received funding from Medical Faculty of LudwigMaximilians-University in Munich, Germany (FöFoLe Reg.Nr. 1089). KR, VB and VLB were funded by the Else Kröner Forschungskolleg. $L L$ received funding from the China Scholarship Council (grant no. 201908080031). PT and SG received a doctoral scholarship from Medical Faculty of Ludwig-Maximilians-University in Munich, Germany. This work was partly supported by the Deutsche Forschungsgemeinschaft (DFG, German Research Foundation, SFBTRR 338/1 2021-452881907 to ST and MS).

\section{Data-sharing statement}

For original data and material, please contact the corresponding authors.

\section{References}

1. Maude SL, Laetsch TW, Buechner J, et al. Tisagenlecleucel in children and young adults with B-cell lymphoblastic leukemia.
N Engl J Med. 2018;378(5):439-448.

2. Locke FL, Ghobadi A, Jacobson CA, et al. Long-term safety and 
activity of axicabtagene ciloleucel in refractory large B-cell lymphoma (ZUMA-1): a single-arm, multicentre, phase 1-2 trial. Lancet Oncol. 2019;20(1):31-42.

3. Schuster SJ, Bishop MR, Tam CS, et al. Tisagenlecleucel in adult relapsed or refractory diffuse large B-cell lymphoma. N Engl $J$ Med. 2019;380(1):45-56.

4. Wang M, Munoz J, Goy A, et al. KTE-X19 CAR T-cell therapy in relapsed or refractory mantle-cell lymphoma. N Engl J Med. 2020;382(14):1331-1342.

5. Shimabukuro-Vornhagen A, Godel P, Subklewe M, et al. Cytokine release syndrome. J Immunother Cancer. 2018;6(1):56.

6. Lee DW, Santomasso BD, Locke FL, et al. ASTCT consensus grading for cytokine release syndrome and neurologic toxicity associated with immune effector cells. Biol Blood Marrow Transplant. 2019;25(4):625-638.

7. Greenbaum U, Strati P, Saliba RM, et al. CRP and ferritin in addition to the EASIX score predict CAR-T-related toxicity. Blood Adv. 2021;5(14):2799-2806.

8. Morris EC, Neelapu SS, Giavridis T, Sadelain M. Cytokine release syndrome and associated neurotoxicity in cancer immunotherapy. Nat Rev Immunol. 2022;22(2):85-96.

9. Brudno JN, Kochenderfer JN. Toxicities of chimeric antigen receptor T cells: recognition and management. Blood. 2016;127(26):3321-3330.

10. Hay KA, Hanafi LA, Li D, et al. Kinetics and biomarkers of severe cytokine release syndrome after CD19 chimeric antigen receptor-modified T-cell therapy. Blood. 2017;130(21):2295-2306.

11. Pennisi M, Sanchez-Escamilla M, Flynn JR, et al. Modified-EASIX predicts severe cytokine release syndrome and neurotoxicity after chimeric antigen receptor (CAR) T cells. Blood Adv. 2021;5(17):3397-3406.

12. Nastoupil LJ, Jain MD, Feng L, et al. Standard-of-care axicabtagene ciloleucel for relapsed or refractory large B-cell lymphoma: results from the US lymphoma CAR T consortium. J Clin Oncol. 2020;38(27):3119-3128.

13. Pasquini MC, Hu ZH, Curran K, et al. Real-world evidence of tisagenlecleucel for pediatric acute lymphoblastic leukemia and non-Hodgkin lymphoma. Blood Adv. 2020;4(21):5414-5424.

14. Neelapu SS, Locke FL, Bartlett NL, et al. Axicabtagene ciloleucel CAR T-cell therapy in refractory large B-cell lymphoma. N Engl J Med. 2017;377(26):2531-2544.

15. Karschnia P, Jordan JT, Forst DA, et al. Clinical presentation, management, and biomarkers of neurotoxicity after adoptive immunotherapy with CAR T cells. Blood. 2019;133(20):2212-2221.

16. Gust J, Hay KA, Hanafi LA, et al. Endothelial activation and blood-brain barrier disruption in neurotoxicity after adoptive immunotherapy with CD19 CAR-T cells. Cancer Discov. 2017;7(12):1404-1419.

17. Santomasso BD, Park JH, Salloum D, et al. Clinical and biological correlates of neurotoxicity associated with CAR T-cell therapy in patients with B-cell acute lymphoblastic leukemia. Cancer Discov. 2018;8(8):958-971.

18. Strati P, Nastoupil LJ, Westin J, et al. Clinical and radiologic correlates of neurotoxicity after axicabtagene ciloleucel in large B-cell lymphoma. Blood Adv. 2020;4(16):3943-3951.

19. Louie JK, Acosta M, Samuel MC, et al. A novel risk factor for a novel virus: obesity and 2009 pandemic influenza A (H1N1). Clin Infect Dis. 2011;52(3):301-312.

20. Kolyva AS, Zolota V, Mpatsoulis D, et al. The role of obesity in the immune response during sepsis. Nutr Diabetes. 2014;4(9):e137.

21. Petronilho F, Giustina AD, Nascimento DZ, et al. Obesity exacerbates sepsis-induced oxidative damage in organs. Inflammation. 2016;39(6):2062-2071.
22. Hotamisligil GS. Inflammation, metaflammation and immunometabolic disorders. Nature. 2017;542(7640):177-185.

23. Ferrante AW, Jr. The immune cells in adipose tissue. Diabetes Obes Metab. 2013;15(3):34-38.

24. Ibrahim MM. Subcutaneous and visceral adipose tissue: structural and functional differences. Obes Rev. 2010;11(1):11-18.

25. Misra A, Vikram NK. Clinical and pathophysiological consequences of abdominal adiposity and abdominal adipose tissue depots. Nutrition. 2003;19(5):457-466.

26. Ansaldo AM, Montecucco F, Sahebkar A, Dallegri F, Carbone F. Epicardial adipose tissue and cardiovascular diseases. Int $\mathrm{J}$ Cardiol. 2019;278:254-260.

27. lacobellis G. Epicardial adipose tissue in endocrine and metabolic diseases. Endocrine. 2014;46(1):8-15.

28. Smith SR, Lovejoy JC, Greenway F, et al. Contributions of total body fat, abdominal subcutaneous adipose tissue compartments, and visceral adipose tissue to the metabolic complications of obesity. Metabolism. 2001;50(4):425-435.

29. Lundbom J, Hakkarainen A, Lundbom N, Taskinen MR. Deep subcutaneous adipose tissue is more saturated than superficial subcutaneous adipose tissue. Int $\mathrm{J}$ Obes (Lond). 2013;37(4):620-622.

30. Nelke C, Dziewas R, Minnerup J, Meuth SG, Ruck T. Skeletal muscle as potential central link between sarcopenia and immune senescence. EBioMedicine. 2019;49:381-388.

31. Rogeri PS, Gasparini SO, Martins GL, et al. Crosstalk between skeletal muscle and immune system: which roles do IL- 6 and glutamine play? Front Physiol. 2020;11:582258.

32. Giudice J, Taylor JM. Muscle as a paracrine and endocrine organ. Curr Opin Pharmacol. 2017;34:49-55.

33. Conlon KC, Lugli E, Welles HC, et al. Redistribution, hyperproliferation, activation of natural killer cells and CD8 T cells, and cytokine production during first-in-human clinical trial of recombinant human interleukin-15 in patients with cancer. J Clin Oncol. 2015;33(1):74-82.

34. Munoz-Canoves P, Scheele C, Pedersen BK, Serrano AL. Interleukin-6 myokine signaling in skeletal muscle: a doubleedged sword? FEBS J. 2013;280(17):4131-4148.

35. Duggal NA, Pollock RD, Lazarus NR, Harridge S, Lord JM. Major features of immunesenescence, including reduced thymic output, are ameliorated by high levels of physical activity in adulthood. Aging Cell. 2018;17(2):e12750.

36. Gomez-Perez SL, Haus JM, Sheean P, et al. Measuring abdominal circumference and skeletal muscle from a single cross-sectional computed tomography image: a step-by-step guide for clinicians using National Institutes of Health ImageJ. JPEN J Parenter Enteral Nutr. 2016;40(3):308-318.

37. Prado CM, Baracos VE, McCargar LJ, et al. Body composition as an independent determinant of 5-fluorouracil-based chemotherapy toxicity. Clin Cancer Res. 2007;13(11):3264-3268.

38. Jayawardena E, Li D, Nakanishi R, et al. Non-contrast cardiac CT-based quantitative evaluation of epicardial and intrathoracic fat in healthy, recently menopausal women: reproducibility data from the Kronos Early Estrogen Prevention Study. J Cardiovasc Comput Tomogr. 2020;14(1):55-59.

39. Jain MD, Zhao $H$, Wang $X$, et al. Tumor interferon signaling and suppressive myeloid cells are associated with CAR T-cell failure in large B-cell lymphoma. Blood. 2021;137(19):2621-2633.

40. Trestini I, Caldart A, Dodi A, et al. Body composition as a modulator of response to immunotherapy in lung cancer: time to deal with it. ESMO Open. 2021;6(2):100095.

41. Cortellini A, Ricciuti B, Tiseo M, et al. Baseline BMI and BMI variation during first line pembrolizumab in NSCLC patients with a PD-L1 expression $\geq 50 \%$ : a multicenter study with 
external validation. J Immunother Cancer. 2020;8(2):e001403.

42. Rogado J, Sanchez-Torres JM, Romero-Laorden N, et al. Immune-related adverse events predict the therapeutic efficacy of anti-PD-1 antibodies in cancer patients. Eur $\mathrm{J}$ Cancer. 2019;109:21-27.

43. Stevenson JKR, Qiao Y, Chan KKW, et al. Improved survival in overweight and obese patients with aggressive B-cell lymphoma treated with rituximab-containing chemotherapy for curative intent. Leuk Lymphoma. 2019;60(6):1399-1408.

44. Scheich S, Enssle JC, Mucke VT, et al. Obesity is associated with an impaired survival in lymphoma patients undergoing autologous stem cell transplantation. PLoS One. 2019;14(11):e0225035.

45. Wudhikarn K, Bansal R, Khurana A, et al. The impact of obesity and body weight on the outcome of patients with relapsed/refractory large B-cell lymphoma treated with axicabtagene ciloleucel. Blood Cancer J. 2021;11(7):124.

46. Wei J, Liu Y, Wang C, et al. The model of cytokine release syndrome in CAR T-cell treatment for B-cell non-Hodgkin lymphoma. Signal Transduct Target Ther. 2020;5(1):134.

47. Rejeski K, Perez A, Sesques P, et al. CAR-HEMATOTOX: a model for CAR T-cell-related hematologic toxicity in relapsed/refractory large B-cell lymphoma. Blood. 2021;138(24):2499-2513.

48. Emmons R, Niemiro GM, De Lisio M. Hematopoiesis with obesity and exercise: role of the bone marrow niche. Exerc Immunol Rev. 2017;23:82-95.
49. Eder K, Baffy N, Falus A, Fulop AK. The major inflammatory mediator interleukin- 6 and obesity. Inflamm Res. 2009;58(11):727-736.

50. Roytblat L, Rachinsky M, Fisher A, et al. Raised interleukin-6 levels in obese patients. Obes Res. 2000;8(9):673-675.

51. Fain JN, Madan AK, Hiler ML, Cheema P, Bahouth SW. Comparison of the release of adipokines by adipose tissue, adipose tissue matrix, and adipocytes from visceral and subcutaneous abdominal adipose tissues of obese humans. Endocrinology. 2004;145(5):2273-2282.

52. Christ A, Latz E. The Western lifestyle has lasting effects on metaflammation. Nat Rev Immunol. 2019;19(5):267-268.

53. Myers GD, Verneris MR, Goy A, Maziarz RT. Perspectives on outpatient administration of CAR-T cell therapy in aggressive Bcell lymphoma and acute lymphoblastic leukemia. J Immunother Cancer. 2021;9(4):e002056.

54. Martin L, Birdsell L, Macdonald N, et al. Cancer cachexia in the age of obesity: skeletal muscle depletion is a powerful prognostic factor, independent of body mass index. $J$ Clin Oncol. 2013;31(12):1539-1547.

55. Cruz-Jentoft AJ, Bahat G, Bauer J, et al. Sarcopenia: revised European consensus on definition and diagnosis. Age Ageing. 2019;48(4):16-31.

56. Rubin DB, Al Jarrah A, Li K, et al. Clinical predictors of neurotoxicity after chimeric antigen receptor T-cell therapy. JAMA Neurol. 2020;77(12):1536-1542. 